\title{
The Efficacy of Auriculotherapy for Smoking Cessation: A Randomized, Placebo-Controlled Trial
}

\author{
Deborah J. Fritz, RN, PhD, Robert M. Carney, PhD, Brian Steinmeyer, MS, \\ Gary Ditson, DC, Nina Hill, RN, MSN, and Joyce Zee-Cheng, RN, MSN
}

Background: Quitting smoking remains a challenge for almost one-third of the military veteran population. Alternatives to pharmacological therapies such as acupuncture, acupressure, and electrical stimulation have received minimal attention in research but have been widely reported to be popular and safe interventions for smoking cessation.

Methods: This randomized, double-blind, placebo-controlled clinical trial of 125 veterans was conducted to determine whether aural electrical stimulation (auriculotherapy) once a week for 5 consecutive weeks is associated with a higher rate of smoking abstinence are than observed with sham stimulation.

Results: Auriculotherapy was found to be safe and largely free from significant side effects. However, there was no difference in the rate of smoking cessation between those participants who received true auriculotherapy and those who received sham auriculotherapy. The auriculotherapy group achieved a rate of $20.9 \%$ abstinence versus $17.9 \%$ for the placebo arm after 6 weeks.

Conclusion: The results of this randomized, controlled clinical trial do not support the use of auriculotherapy to assist with smoking cessation. It is possible that a longer treatment duration, more frequent sessions, or other modifications of the intervention protocol used in this study may result in a different outcome. However, based on the results of this study, there is no evidence that auriculotherapy is superior to placebo when offered once a week for 5 weeks, as described in previous uncontrolled studies. (J Am Board Fam Med 2013;26:61-70.)

Keywords: Alternative Medicine, Tobacco Cessation, Veterans

Smoking is a major health hazard. Although the risk of smoking-related diseases and death decline sharply when smokers quit, 19\% of Americans currently smoke. ${ }^{1}$ Rates of smoking are even higher among US military veterans, with about 1 in 3 current smokers. ${ }^{2}$ In keeping with the 2008 clinical guidelines for smoking cessation, veterans are asked

This article was externally peer reviewed.

Submitted 7 June 2012; revised 7 October 2012; accepted 10 October 2012.

From the Veteran's Administration (DF, NG, JZ) and Department of Psychiatry, Washington University (RMC, BS), St. Louis, MO; and private practice in Wentzville, MO (GD).

Funding: This material is based on work supported by the Office of Research and Development, Health Services Research and Development Service, Department of Veterans Affairs and is funded by Veterans Administration Nurse Research Initiative NRI-08 371.

Conflict of interest: none declared.

Corresponding author: Deborah Fritz RN, PhD, Veteran's Administration, St. Louis, 915 North Grand Blvd, St. Louis, MO 63106 (E-mail: deborah.fritz@va.gov). about their smoking status annually and are offered group or individual counseling and either bupropion or nicotine replacement therapy to help them achieve smoking abstinence. ${ }^{3,4}$

Both bupropion and nicotine replacement therapy have been found to be more effective than placebo or no treatment, ${ }^{5,6}$ but they are ineffective in many cases. In fact, a meta-analysis of clinical trials found that bupropion and a nicotine patch result in abstinence in only $20 \%$ and $13 \%$ of participants, respectively. ${ }^{7}$ At the Veterans Affairs Medical Center (VAMC), varencline, a second-line medication with a reported $25 \%$ abstinence rate, is considered only after bupropion or nicotine replacement therapy or a combination of the two have failed, and then only after appropriate psychiatric and cardiovascular screening. ${ }^{7-12}$

Alternatives to pharmacological therapies such as acupuncture, acupressure, and electrical stimulation have received minimal attention in the re- 
search but are used widely, and proponents of their use believe them to be effective and safe interventions for smoking. ${ }^{13}$ However, a recent Cochrane review found little evidence from randomized controlled trials that any of these therapies work better than placebo. ${ }^{14}$ Auriculotherapy is similar to acupuncture in many respects, but rather than using traditional needles it relies on an undetectable electrical current to stimulate prescribed points on the ear that are thought to facilitate smoking cessation. Although widely used, there is limited evidence to support its efficacy in smoking cessation. In one study, 27 participants received auriculotherapy treatments weekly for 5 weeks for smoking cessation and attained a $77 \%$ abstinence rate at a 3 -month follow-up. ${ }^{15}$ However, this study was not a randomized trial, and cessation rate was determined only by self-report. Antoniou and colleagues ${ }^{16}$ applied auriculotherapy to acupuncture points on both ears on one occasion using either 12 to $20 \mathrm{mAmp}$ (active) or $0 \mathrm{mAmp}$ (placebo). Although there was no difference between groups, study participants achieved a $20.6 \%$ cessation rate 12 months after treatment.

Previous studies of these therapies have significant methodological limitations, including small sample sizes, nonrandomization, nonblinded assessment, high dropout rates, and outcome assessments that rely only on self-report. ${ }^{14}$ Thus, there is a clear need for controlled, carefully conducted trials to establish the efficacy of these nonpharmacologic smoking interventions.

This randomized, double-blind, placebo-controlled clinical trial was conducted to determine whether smoking abstinence rates were higher among a military veteran population receiving auriculotherapy once a week for 5 consecutive weeks compared with sham stimulation. The study hypothesis was that auriculotherapy would be superior to placebo for achieving smoking cessation.

\section{Methods}

\section{Recruitment and Eligibility Screening}

Participants were recruited between August 2010 and January 2011 at the St. Louis Veterans Affairs Medical Center. The inclusion criteria were age 19 years or older and currently smoking $\geq 10$ cigarettes per day. Study exclusions included (1) pathologic condition of the ear; (2) pregnancy; (3) indwelling cardiac pacemaker or defibrillator; (4) actively psychotic, severe cognitive impairment, or current significant substance abuse disorder; and (5) currently receiving another intervention for smoking cessation.

All potential participants were required to attend a minimum of one "stop smoking" class, which is offered routinely at the VA. This 1-hour "First Step" class teaches smokers how to prepare for their quit date and how to cope with situations that may increase the risk of relapse. Attendance at the smoking cessation class assured that all participants had the same information regarding the importance of smoking cessation and what to expect during the process, as well as knowledge of some of the more useful behavioral strategies for effective smoking cessation.

Participants who provided written, informed consent, completed a 1-hour "stop smoking class," scored $<20$ on the 9-item Patient Health Questionnaire (PHQ-9) ${ }^{17}$ and had a urine cotinine value higher than level $3(\geq 200 \mathrm{ng} / \mathrm{mL})^{18}$ were enrolled.

\section{Study Design}

This is a randomized, double-blind, placebo-controlled clinical trial for smoking cessation in a population of US military veterans. It is designed to determine the efficacy of auriculotherapy (stimulation of predetermined bilateral auricle points) by comparing the standard intervention $(80 \mathrm{~Hz})$ with a sham placebo $(0 \mathrm{~Hz})$. All participants were scheduled for 5 individual treatments, each 1 week apart, using the Stim Flex 400A transcutaneous electrical nerve stimulation unit (Electro Medical Inc., Tulsa, OK). The stimulation sites on the ear-known in acupuncture as Lung, Shen Men, Nicotine, Point Zero, and Palate-were used in this study, as recommended by auricular acupuncture studies ${ }^{19,20}$ and the guidelines provided by the Auriculotherapy Manual. ${ }^{21}$ Military veterans $(\mathrm{N}=125)$ were randomized to either the active or placebo condition. Written, informed consent approved by the VA Human Subjects Committee was obtained before randomization.

\section{Prerandomization Phase}

Demographic characteristics, smoking history, and urine cotinine levels were determined at baseline. The Fagerström Test of Nicotine Dependence, ${ }^{22}$ the stage of change (Transtheoretical model), ${ }^{23}$ PHQ-9, ${ }^{17}$ 4-item Perceived Stress Scale, ${ }^{24}$ and the 
Minnesota Withdrawal Scale ${ }^{25}$ questionnaires were administered before randomization.

\section{Randomization}

Permuted block assignments were generated by the study statistician (BS) from a random-number generator in advance of the trial's start date. Treatment assignment was indicated inside sealed, sequentially numbered opaque envelopes, opened by the primary investigator (DF) at the time of randomization.

\section{Treatment and Follow-up}

Two registered nurses were certified in the application of the Stim Flex 400A auriculotherapy device by one of the investigators (GD), who is nationally recognized as a certified and experienced practitioner and teacher of acupuncture and auriculotherapy. The nurses received ongoing supervision, and more than $25 \%$ of the study treatments were observed directly by the investigator to ensure consistent and appropriate application of the treatment over time.

All interventions comprised 5 weekly, 20-minute sessions. Two Stim Flex 400A units were used. One unit was a usual functioning machine (active) and the second was altered to disable the treatment electrical current to prevent flow to the probe (placebo). Both machines used identical probes with the ability to sense the prescribed sites and were identical in appearance, sound, and operation. All personnel with patient access remained blinded to which machine had been altered and which provided the stimulation.

The double-blind condition was maintained throughout the data collection period. Only the study statistician and members of the Data and Safety Monitoring Committee were unblinded to treatment assignment during the trial, and they had no contact with study personnel. At the end of the treatment schedule, study participants were asked whether they thought they had received the active treatment during the course of the trial. After 2 and 5 treatments (weeks 3 and 6), smoking abstinence, perceived stress, and nicotine withdrawal symptoms were reassessed.

\section{Study Outcomes}

Abstinence was determined at weeks 3 and 6 by patient self-report of smoking (yes/no) and urine cotinine level $(<200 \mathrm{ng} / \mathrm{mL})^{26}$ from the NicAlert point of care test, which reflects smoking status over a period of 2 to 3 days. The method has a sensitivity of $100 \%$ and a specificity of $95 \%$ for identifying patients who smoked even a single cigarette in the previous 3 days. ${ }^{27}$ Other outcomes included (1) nicotine withdrawal symptoms (Minnesota Withdrawal Scale score before and after treatment); (2) perceived stress (4-item Perceived Stress Scale score before and after treatment), and (3) self-reported frequency of tobacco use.

\section{Statistical Analysis}

$\chi^{2}$ Tests and analysis of variance were used to compare demographic, medical, and smoking characteristics at baseline between the treatment groups. Fisher exact tests were reported when expected cell frequencies were too small for a valid $\chi^{2}$ test.

A 2-proportion $z$ test was used to formally test whether the proportion of abstinence differed between groups at weeks 3 and 6 . In a second step, a logistic regression model was used to test the treatment effect while controlling for other baseline prognostic variables: patient age; sex; education; comorbid anxiety, depression, posttraumatic stress disorder; number of cigarettes smoked per day; stage of change; and perceived stress. The treatment factor odds ratio (OR) reflects the odds associated with abstinence in the treated group compared with the control group and provides a test of the treatment effect in all fitted logistic regression models.

The other interval-scaled outcomes were fitted to a linear mixed effects model to test whether there was a treatment effect over time and to account for intrasubject variation from repeated measurements. In unadjusted analyses, each outcome variable was regressed on a between-subjects factor for treatment $(\mathrm{G})$, a within-subjects factor of time (weeks; $\mathrm{T}$ ), the interaction between treatment and time $(\mathrm{G} \times \mathrm{T})$, and the value of the outcome before measurement. In subsequent adjusted analyses, we controlled for self-reported motivation to quit smoking and nicotine dependence at baseline. Weighted $\kappa$ was used to examine the overall and treatmentstratified level of agreement between the result of the NicAlert test and self-reported abstinence at weeks 3 and 6 .

To provide conservative estimates of the treatment effect and valid inference for the intent-totreat (ITT) population, ${ }^{28}$ multiple imputation ${ }^{29}$ 
was used to address missing follow-up data due to attrition. We created 20 multiply imputed datasets from an imputer's model that comprised both analysis model variables and auxiliary variables that were likely correlates of the missing data mechanism. The auxiliary variables help to strengthen the missing at random assumption needed to allow for valid ITT inference. The ITT analyses represent the primary analysis; however, as part of a secondary analysis, we repeated the analysis with a subgroup of patients who had complete follow-up data (ie, "completers"). The target sample size was based on a medium to large hypothesized effect for auriculotherapy. The planned sample size provided more than $80 \%$ power to detect a medium to large (20\% to $25 \%$ ) auriculotherapy effect in the target population.

Each statistical model was examined for any serious model violations. For the logistic regression and mixed models, we examined the fitted residuals to assess potential outliers and any influential observations and examined the overall fit of each model. All statistical tests were 2-sided, and statistical significance was deemed as $P<.05$, using SAS 9.2 statistical software (SAS Institute, Inc., Cary, NC).

\section{Results}

Two hundred thirteen veterans who either responded to a mailing or were referred by their primary care physician expressed interest in the study (Figure 1). Of these, 17 were excluded because they did not meet eligibility criteria. The remaining 196 were scheduled to attend a 1-hour "stop smoking" class offered at the VA. Twentynine did not attend the mandatory smoking class. After completion of the class, participants were given an appointment for enrollment and a treatment schedule. Eleven did not keep the consent appointment. Another 31 did not enroll for other reasons. Thus, 125 participants were randomized: 64 to the intervention group and 61 to the placebo group. No participants in the intervention group dropped out of treatment. However, 6 (9.4\%) had unexplained missed appointments. Four participants in the placebo group (6.6\%) dropped out for personal reasons, including job reassignment, and 4 $(6.6 \%)$ had unexplained missed appointments.

\section{Baseline Characteristics}

The demographic and medical attributes, smoking history, nicotine dependence, and other baseline characteristics of the 2 treatment groups were compared (Table 1). There were no significant differences between the groups except that the intervention group reported higher motivation to quit smoking $(P=.003)$. Twenty-five percent of participants were women.

Participants smoked an average of 30 cigarettes per day, with all but 10 participants (18\%) in the contemplation or preparation stage of change. ${ }^{23}$ Four participants reported low, 94 reported medium, and 25 reported high nicotine dependence on the Fagerström Test of Nicotine Dependence questionnaire. $^{22}$ The groups did not differ on the PHQ-9 depression screening instrument; mean scores for both groups were consistent with mild depression.

\section{Adberence to Treatment Regimen}

Forty-five of the participants assigned to the active group (70\%) and 40 of those assigned to the placebo group (66\%) attended all 5 treatment sessions $(P=.13)$. Of all participants, $85 \%$ received at least 4 treatments (Table 2). Fifty-three of the participants in the active group (83\%) and 52 participants in the placebo group (85\%) completed the questionnaire surveys at baseline and weeks 3 and 6 . There were no between-group differences in satisfaction with the treatments.

The double-blind condition was maintained with the primary investigator, the other investigators, and research staff. However, more patients in the active group (67\%) believed they received the intervention than those in the placebo group (46\%) $(P=.03)$ at week 6.

\section{Outcomes After Treatment}

Self-reported abstinence rates were generally lower than cotinine-defined $(<200 \mathrm{ng} / \mathrm{mL})$ rates, but the intervention and control groups did not differ on the former $(12.3 \%$ and $12.9 \%$, respectively; $P=$ $.93)$ or the latter $(22.3 \%$ and $17.0 \%$, respectively; $P=.50)$ at week 3 in unadjusted analyses (Table 3 ). These findings were consistent after controlling for prognostic baseline variables. Findings between groups on self-reported and cotinine-defined abstinence rates were similar at week 6 in both unadjusted and adjusted analyses. 
Figure 1. Participant flow (ITT, intent to treat).

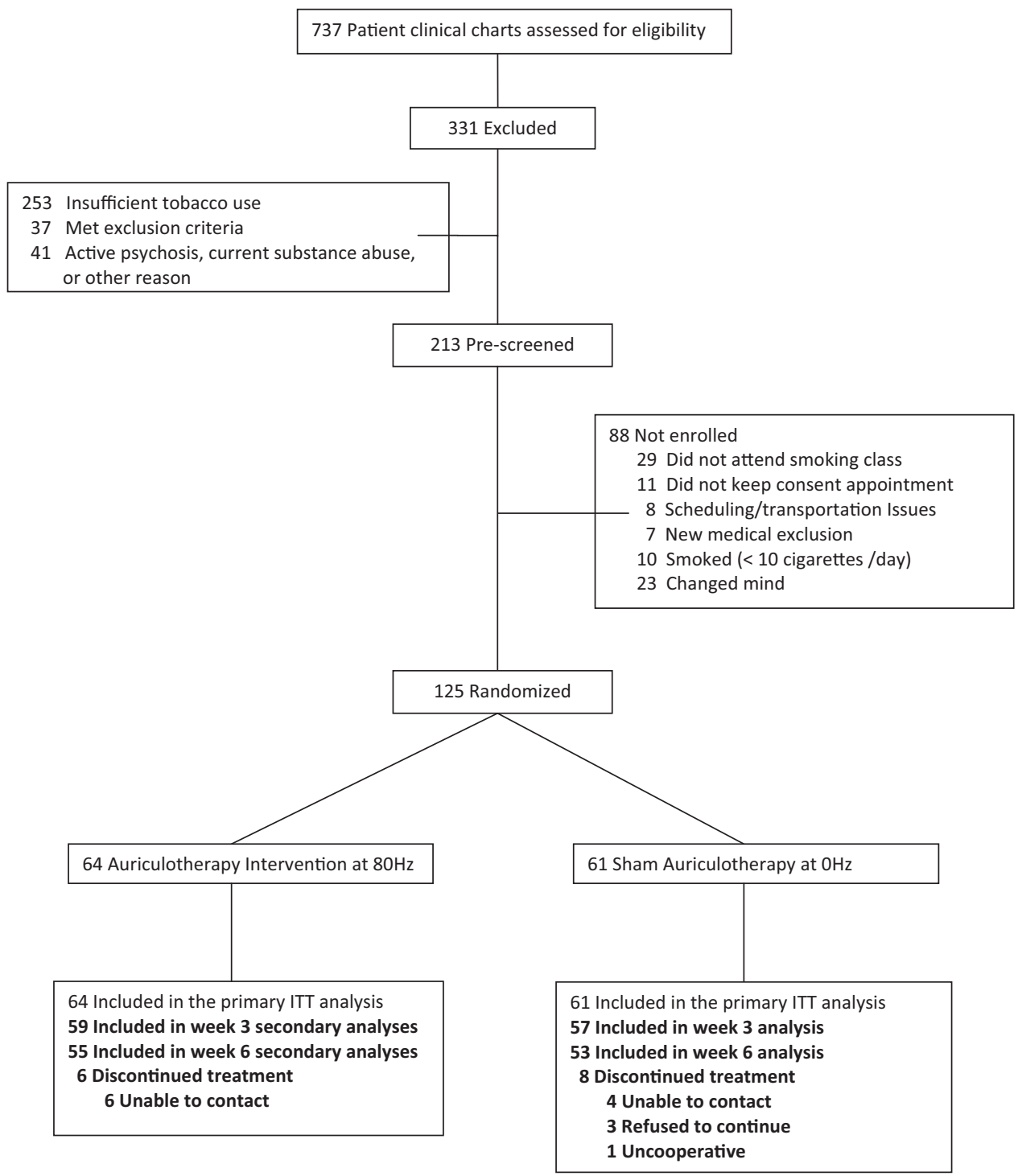

In addition, there was no evidence of any between-group differences over time on the following interval-scaled outcomes in unadjusted analyses: nicotine withdrawal $(P=.42)$, perceived stress $(P=$ $.75)$, or tobacco use $(P=.73)$ (Table 4$)$. These findings did not differ when we controlled for selfreported motivation to quit smoking and nicotine dependence at baseline.

In a post hoc analysis, we examined the level of overall and treatment-stratified agreement between cotinine-defined abstinence and self-reported abstinence. There was substantial agreement between the 2 abstinence outcomes at weeks 3 and $6\left(\kappa_{3}=\right.$ 0.61 and $\kappa_{6}=0.59$, respectively). Agreement between groups depended on the time of measurement. For example, the control group had higher agreement $\left(\kappa_{\mathrm{C}}=0.78\right)$ compared with the intervention group $\left(\kappa_{\mathrm{A}}=0.47\right)$ at week 3 , but at week 6 , the intervention group $\left(\kappa_{\mathrm{A}}=0.67\right)$ had higher level of agreement compared with the control group $\left(\kappa_{\mathrm{C}}=0.51\right)$.

Among those who quit smoking, at week 6, women $(P=.02)$ and all those currently employed $(P=.014)$ were more likely to have achieved abstinence. Those who quit had significantly fewer complaints of withdrawal symptoms $(P=.033)$. In addition, those with a comorbid diagnosis of cancer were more likely to quit smoking $(P=.02)$.

As part of a secondary analysis, we compared the results from the total sample ITT analysis with the results from the participants who actually completed the protocol. The results did not differ be- 
Table 1. Baseline Medical, Smoking, and Demographic Characteristics by Treatment Group $(\mathrm{N}=125)$

\begin{tabular}{|c|c|c|c|}
\hline \multirow[b]{2}{*}{ Characteristics } & \multicolumn{2}{|c|}{ Treatment Group } & \multirow[b]{2}{*}{$P$} \\
\hline & Control $(\mathrm{n}=61)$ & Intervention $(\mathrm{n}=64)$ & \\
\hline \multicolumn{4}{|l|}{ Demographics } \\
\hline Sex (\% female) & $14(23.0)$ & $16(25.0)$ & .79 \\
\hline Age (years) & $55.3 \pm 9.1$ & $55.8 \pm 10.0$ & .81 \\
\hline Race & & & $.67 *$ \\
\hline 1 (African-American) & $42(68.9)$ & $38(59.4)$ & \\
\hline 2 (American Indian) & $0(0.0)$ & $0(0.0)$ & \\
\hline 3 (Asian-American) & $1(1.6)$ & $1(1.6)$ & \\
\hline 4 (Hispanic) & $0(0.0)$ & $1(1.6)$ & \\
\hline 5 (Non-Hispanic white) & $18(29.5)$ & $24(37.5)$ & \\
\hline 6 (Other) & $0(0.0)$ & $0(0.0)$ & \\
\hline Married & $22(36.1)$ & $26(40.6)$ & .60 \\
\hline Education $(>12$ years) & $56(91.8)$ & $58(90.6)$ & .82 \\
\hline Currently employed & $18(30.0)$ & $16(25.8)$ & .61 \\
\hline \multicolumn{4}{|l|}{ Medical } \\
\hline General health rating & & & $.87^{*}$ \\
\hline 0 (Poor) & $6(10.0)$ & $9(14.1)$ & \\
\hline 1 (Good) & $51(85.0)$ & $52(81.3)$ & \\
\hline 2 (Excellent) & $3(5.0)$ & $3(4.7)$ & \\
\hline \multicolumn{4}{|l|}{ Comorbidities } \\
\hline Anxiety & $5(8.2)$ & $10(16.4)$ & .17 \\
\hline Asthma & $9(14.8)$ & $9(15.0)$ & .97 \\
\hline CAD & $11(18.0)$ & $9(14.8)$ & .62 \\
\hline Cancer & $5(8.2)$ & $3(4.9)$ & $.72^{*}$ \\
\hline COPD & $9(14.8)$ & $9(15.0)$ & .97 \\
\hline Depression & $17(27.9)$ & $25(41.0)$ & .13 \\
\hline Diabetes & $20(32.8)$ & $19(31.2)$ & .85 \\
\hline PTSD & $6(9.8)$ & $11(18.3)$ & .18 \\
\hline Other & $12(19.7)$ & $15(24.6)$ & .51 \\
\hline \multicolumn{4}{|l|}{ Smoking history } \\
\hline Age started smoking (years) & $16.8 \pm 4.1$ & $17.6 \pm 5.1$ & .34 \\
\hline Years smoked & $35.4 \pm 13.0$ & $34.2 \pm 12.6$ & .62 \\
\hline Packs per day & $1.7 \pm 3.9$ & $1.8 \pm 5.3$ & .89 \\
\hline Times quit for $>24$ hours during the past month & $0.5 \pm 1.1$ & $0.9 \pm 1.5$ & .14 \\
\hline Has a spouse or significant other who smokes & $16(30.8)$ & $25(45.4)$ & .12 \\
\hline \multicolumn{4}{|l|}{ Questionnaires } \\
\hline Transtheoretical Model of Change category & & & .60 \\
\hline 1 (Precontemplation) & $4(7.3)$ & $6(10.5)$ & \\
\hline 2 (Contemplation) & $21(38.2)$ & $17(29.8)$ & \\
\hline 3 (Preparation) & $30(54.6)$ & $34(59.7)$ & \\
\hline Fagerström Test of Nicotine Dependence & & & $.19^{*}$ \\
\hline 1 (Low) & $0(0.0)$ & $4(6.3)$ & \\
\hline 2 (Medium) & $47(79.7)$ & $47(73.4)$ & \\
\hline 3 (High) & $12(20.3)$ & $13(20.3)$ & \\
\hline Motivation to quit smoking (on a scale of $1-10$ ) & $7.5 \pm 2.4$ & $8.6 \pm 1.7$ & .003 \\
\hline Minnesota Nicotine Withdrawal Scale Score & $19.0 \pm 10.2$ & $19.6 \pm 10.9$ & .75 \\
\hline Patient Health Questionnaire Score & $5.1 \pm 4.5$ & $5.9 \pm 5.5$ & .36 \\
\hline Perceived Stress Scale Score & $5.4 \pm 2.8$ & $5.8 \pm 2.9$ & .45 \\
\hline
\end{tabular}

Continuous variables are reported as (mean \pm standard deviation). Categorical variables represent number of patients (\%). *Fisher exact test.

CAD, coronary artery disease; COPD, chronic obstructive pulmonary disease; PTSD, posttraumatic stress disorder. 
Table 2. Premature Study Termination, Patient Safety, and Treatment Compliance by Treatment Group $(\mathrm{N}=125)$

\begin{tabular}{|c|c|c|c|}
\hline \multirow[b]{2}{*}{ Characteristic } & \multicolumn{2}{|c|}{ Treatment Group } & \multirow[b]{2}{*}{$P$} \\
\hline & Control $(\mathrm{n}=61)$ & Intervention $(\mathrm{n}=64)$ & \\
\hline \multicolumn{4}{|l|}{ Premature study termination } \\
\hline Number reported & $8(13.1)$ & $6(9.4)$ & .51 \\
\hline Primary reason & & & $.12^{*}$ \\
\hline A (Unable to contact) & $4(6.6)$ & $6(9.4)$ & \\
\hline B (Dropped out) & $3(4.9)$ & $0(0.0)$ & \\
\hline C (Uncooperative or nonadherent) & $1(1.6)$ & $0(0.0)$ & \\
\hline \multicolumn{4}{|l|}{ Patient safety } \\
\hline Reported study-related serious AEs (n) & 2 & 2 & \\
\hline No & 2 & 2 & \\
\hline Yes & 0 & 0 & \\
\hline Unknown & 0 & 0 & \\
\hline \multicolumn{4}{|l|}{ Reported study-related AEs (n) } \\
\hline No & 6 & 5 & \\
\hline Yes & 0 & 1 & \\
\hline Unknown & 3 & 0 & \\
\hline \multicolumn{4}{|l|}{ Treatment compliance } \\
\hline Treatments received during the study & & & $.13^{*}$ \\
\hline 0 & $1(1.6)$ & $0(0.0)$ & \\
\hline 1 & $4(6.6)$ & $1(1.6)$ & \\
\hline 2 & $3(4.9)$ & $2(3.1)$ & \\
\hline 3 & $1(1.6)$ & $7(10.9)$ & \\
\hline 4 & $12(19.7)$ & $9(14.1)$ & \\
\hline 5 (All) & $40(65.6)$ & $45(70.3)$ & \\
\hline Surveys completed during the study & & & $.93^{*}$ \\
\hline 0 & $0(0.0)$ & $0(0.0)$ & \\
\hline 1 & $3(4.9)$ & $3(4.7)$ & \\
\hline 2 & $6(9.8)$ & $8(12.5)$ & \\
\hline 3 (All) & $52(85.3)$ & $53(82.8)$ & \\
\hline${ }^{1}$ Patient satisfaction with control/auriculotherapy & & & $.13^{*}$ \\
\hline 1 (Waste of time) & $3(5.9)$ & $1(1.8)$ & \\
\hline 2 (Poor) & $4(7.8)$ & $2(3.6)$ & \\
\hline 3 (Fair) & $11(21.6)$ & $10(18.2)$ & \\
\hline 4 (Good) & $10(19.6)$ & $23(41.8)$ & \\
\hline 5 (Excellent) & $23(45.1)$ & $19(34.6)$ & \\
\hline Patient recommends auriculotherapy for family/friends ${ }^{\dagger}$ & $46(75.4)$ & $52(81.3)$ & .45 \\
\hline
\end{tabular}

Continuous variables are reported as (mean $\pm \mathrm{SD})$; categorical variables represent number of patients (\%).

*Fisher exact test.

${ }^{\dagger}$ Of the 125 randomized participants, 89 provided study satisfaction and blinding assessment data at week 6.

$\mathrm{AE}$, adverse event.

tween the total sample and the protocol completers (data not shown).

\section{Adverse Events}

Adverse events were monitored weekly by the nurses who administered the intervention. There were a total of 15 adverse events ( 9 intervention, 6 placebo) reported (Table 2). None of the serious adverse events were deemed by the Data and Safety Monitoring Committee to be related to the study protocol, and only one less serious event (auricle discomfort without redness or swelling) was found to be related to the intervention.

\section{Discussion}

To our knowledge, this is the largest and most rigorous trial to date to investigate the safety, acceptability, and efficacy of auriculotherapy for 
Table 3. Effect of Auriculotherapy on Self-Reported and Cotinine-Defined Abstinence at Weeks 3 and 6

\begin{tabular}{|c|c|c|c|}
\hline \multirow[b]{2}{*}{ Outcome Measure } & \multicolumn{2}{|c|}{ Treatment Group } & \multirow[b]{2}{*}{$P$} \\
\hline & Control $(\mathrm{n}=61)$ & Intervention $(\mathrm{n}=64)$ & \\
\hline \multicolumn{4}{|c|}{ Self-reported abstinence } \\
\hline Week $3^{*}$ & $12.9(4.0-21.7)$ & $12.3(4.0-20.7)$ & .93 \\
\hline Week $3^{+}$ & 1.0 & $0.81(0.23-2.83)$ & .74 \\
\hline Week $6^{*}$ & $17.9(7.2-28.6)$ & $20.9(10.2-31.6)$ & .69 \\
\hline Week $6^{+}$ & 1.0 & $1.19(0.40,3.53)$ & .75 \\
\hline \multicolumn{4}{|c|}{ Cotinine-defined $(<200 \mathrm{ng} / \mathrm{mL})$ abstinence } \\
\hline Week $3^{*}$ & $17.0(6.9-27.2)$ & $22.3(11.0-33.6)$ & .50 \\
\hline Week $3^{\dagger}$ & 1.0 & $1.44(0.48-4.36)$ & .51 \\
\hline Week $6^{*}$ & $24.6(12.2-37.0)$ & $20.5(9.9-31.0)$ & .61 \\
\hline Week $6^{\dagger}$ & 1.0 & $0.75(0.28-2.01)$ & .56 \\
\hline
\end{tabular}

*Values represent the proportion (95\% confidence interval) of patients who were abstinent in each group.

${ }^{\dagger}$ Abstinence (yes/no) was regressed in the treatment group and for the following covariates in logistic regression models: patient age, sex, education, comorbid anxiety, depression, posttraumatic stress disorder, number of cigarettes smoked per day at baseline, model of change category, and perceived stress scale score. Values are shown as odds ratio (95\% confidence interval) estimates and represent the odds of abstinence in the auriculotherapy group compared with the control group.

smoking cessation. We found auriculotherapy to be safe and largely free from significant side effects. We had no difficulty in recruiting participants for this study, and the dropout rate was acceptably low. In fact, most participants were highly motivated to quit smoking, and most found auriculotherapy to be an acceptable method for assisting their efforts. However, the study found no difference in the rate of smoking cessation between those participants who received true and those who received sham auriculotherapy after 3 and 6 weeks of treatment. Furthermore, whereas some uncontrolled studies have found auriculotherapy to have an impact on withdrawal symptoms in the short term, there was no evidence of any between-group differences over time regarding nicotine withdrawal symptoms in this study.

A higher proportion of participants in the true auriculotherapy group compared with the sham control group correctly guessed their group assignment. Given the efforts to maintain blinded treatment, this was unexpected. The actual current generated by the auriculotherapy procedure is considered to be below the threshold for human detection. It also was noted that a nearly identical proportion of participants in both groups requested a decrease in the level of current during treatments, complaining that it was slightly uncomfortable. Furthermore, at the end of the study, the registered nurses who administered the auriculotherapy treatments reported that they did not know which Stim Flex 400 provided a current during the treatment, and they guessed at a chance level. Thus, it is unlikely that the nurses unwittingly communicated to the participants that they received the real or the sham intervention. In any case, there was no clear effect of this on the outcome of the study.

The comparison of true with sham auriculotherapy was intended to differentiate the putative physiologic effects of the intervention from the placebo effects of the procedure itself. The inability to provide a true placebo condition has been a concern in the literature. To maintain the doubleblind condition, both Stim Flex machines had a minute amount of current necessary to sense the auriculotherapy points, and pressure was applied to each point with the probe, although without electrical current in the sham condition. It is possible that the pressure alone applied to these points provided a therapeutic effect and therefore was not a true placebo condition. However, abstinence rates in both arms were considerably smaller than those reported in uncontrolled studies.

An alternative placebo intervention was used by Yeh et $\mathrm{al},{ }^{30}$ who applied low-frequency electrical acupuncture for 20 minutes once a week with sham acupoints $5 \mathrm{~mm}$ apart from true points. As in our study, they found no statistically significant differences between the 2 groups. However, because of the sensitivity of auricular points in close proximity to each other, it is possible that simply touching these points without electrical stimulation may provide a therapeutic effect. 
Table 4. Effect of Auriculotherapy on Interval-Scaled Nicotine Withdrawal, Perceived Stress, and Tobacco Use at Weeks 3 and 6

\begin{tabular}{|c|c|c|c|c|c|}
\hline \multirow[b]{2}{*}{ Outcome Measure } & \multicolumn{2}{|c|}{ Treatment Group } & \multicolumn{3}{|c|}{${ }^{4} P^{*}$} \\
\hline & Control $(\mathrm{n}=61)$ & Intervention $(\mathrm{n}=64)$ & G & $\mathrm{T}$ & $\mathrm{G} \times \mathrm{T}$ \\
\hline \multicolumn{6}{|c|}{ Minnesota Nicotine Withdrawal Scale score } \\
\hline \multicolumn{6}{|c|}{ Unadjusted mixed model $^{\dagger}$} \\
\hline Week 3 & $14.7(12.7-16.7)$ & $13.4(11.4-15.3)$ & .56 & .06 & .42 \\
\hline Week 6 & $15.6(13.5-17.7)$ & $15.5(13.5-17.5)$ & & & \\
\hline \multicolumn{6}{|c|}{ Adjusted mixed model ${ }^{\ddagger}$} \\
\hline Week 3 & $14.4(12.2-16.8)$ & $12.9(10.7-15.1)$ & .46 & .10 & .58 \\
\hline Week 6 & $15.4(13.0-17.8)$ & $14.8(12.5-17.1)$ & & & \\
\hline \multicolumn{6}{|c|}{ Perceived Stress Scale score } \\
\hline \multicolumn{6}{|c|}{ Unadjusted mixed model $^{\dagger}$} \\
\hline Week 3 & $5.2(4.5-5.9)$ & $4.8(4.1-5.5)$ & .42 & .05 & .75 \\
\hline Week 6 & $5.6(4.9-6.3)$ & $5.3(4.6-6.0)$ & & & \\
\hline \multicolumn{6}{|c|}{ Adjusted mixed model ${ }^{\ddagger}$} \\
\hline Week 3 & $5.0(4.3-5.8)$ & $4.8(4.0-5.5)$ & .61 & .16 & .99 \\
\hline Week 6 & $5.4(4.6-6.2)$ & $5.1(4.4-5.9)$ & & & \\
\hline \multicolumn{6}{|c|}{ Cigarettes smoked among nonabstinent patients ${ }^{\S}(\mathrm{n})$} \\
\hline \multicolumn{6}{|c|}{ Unadjusted mixed model ${ }^{\dagger}$} \\
\hline Week 3 & $9.5(6.7-12.2)$ & $6.8(4.1-9.5)$ & .17 & .34 & .73 \\
\hline Week 6 & $10.0(7.3-12.7)$ & $8.0(5.3-10.7)$ & & & \\
\hline \multicolumn{6}{|c|}{ Adjusted mixed model ${ }^{\ddagger}$} \\
\hline Week 3 & $7.9(4.9-10.9)$ & $7.0(3.9-10.1)$ & .76 & .29 & .79 \\
\hline Week 6 & $8.7(5.7-11.7)$ & $8.3(5.2-11.4)$ & & & \\
\hline
\end{tabular}

*Reported $P$ values for each measure represent overall tests about type 3 fixed effects for both unadjusted and adjusted models. ${ }^{\dagger}$ Values represent least squares mean (95\% confidence interval) estimates from an unadjusted mixed model in which each outcome was regressed for treatment group $(\mathrm{G})$, time occasion $(\mathrm{T})$, the interaction between group and time $(\mathrm{G} \times \mathrm{T})$, and the value of the outcome measure before measurement.

${ }^{\ddagger}$ Values represent least squares mean (95\% confidence interval) estimates from an adjusted mixed model that controls for age, sex, and the motivation to quit smoking at baseline.

${ }^{\S}$ Study participants that remained nonabstinent at weeks 3 and 6 , according to urine cotinine levels.

In addition, the protocol used in this study may not have been optimal to achieve smoking cessation. However, although it is possible that using other stimulation frequencies or intensities, longer durations of electrical stimulation, or more frequent or longer treatment sessions may have produced a different outcome, we used the existing literature and the manufacturer's recommendations when designing this protocol. Furthermore, we chose to provide weekly treatments for 5 weeks based on the only published study using the Stim Flex machine. ${ }^{13}$

The results of the trial are consistent with the conclusion of the Cochrane review of studies of acupuncture and related interventions for smoking cessation. The report found "little evidence from randomized controlled trials that any of these therapies work better than placebo," ${ }^{14}$ despite widespread use and positive anecdotal reports. This study's findings also are consistent with 2 previous studies that used variations of the auriculotherapy methodology. ${ }^{16,30}$

\section{Conclusion}

We found auriculotherapy to be a safe and acceptable procedure to assist with smoking cessation among the US military veterans who participated in this study. However, there were no differences in the rate of smoking cessation after 3 and 6 weeks of treatment between those who received the true versus sham auriculotherapy intervention. Although it is possible that modifications of the intervention protocol used in this study may have resulted in a different outcome, the results of this randomized, controlled clinical trial do not support the use of auriculotherapy to assist smoking cessation. 


\section{References}

1. CDC, Adult cigarette smoking in the United States: Current estimate. http:www.cdc.gov/tobacco/data statistics/fact_sheets/adult_data/cig_smoking/index. htm. Accessed November 11, 2012.

2. Department of Veteran Affairs. VHA survey of veteran enrollees' health and reliance upon VA; 2011. Available from: http://www.va.gov/ HEALTHPOLICYPLANNING/SOE2011/SoE 2011_Report.pdf. Accessed September 2012.

3. US Department of Health and Human Services. Treating tobacco use and dependence: 2008 update. www.ahrq.gov/path/tobacco.htm. Accessed November 3, 2012.

4. Cunningham CL, Kaboli P, Ono S, Vander Weg MW. A qualitative evaluation of knowledge of and attitudes toward VA smoking cessation services. J Smoke Cessat 2011;6:152-8.

5. Fossati R, Apolone G, Negri E, et al. A double-blind, placebo-controlled, randomized trial of bupropion for smoking cessation in primary care. Arch Intern Med 2007;167:1791-7.

6. Myung SK, Yoo KY, Oh E, et al. Meta-analysis of studies investigating one-year effectiveness of transdermal nicotine patches for smoking cessation. Am J Health Syst Pharm 2007;64:2471-6.

7. Eisenberg MJ, Filion KB, Yavin D, et al. Pharmacotherapies for smoking cessation: a meta-analysis of randomized controlled trials. CMAJ. 2008;179:135-44.

8. Veterans Administration. Varenicline criteria for prescribing, July 2011. Available from: http:// www.healthquality.va.gov/tuc/VareniclineCriteriafor Prescribing.pdf. Accessed September 21, 2012.

9. Tonstad S, Davies S, Flammer M, Russ C, Hughes $\mathrm{J}$. Psychiatric adverse events in randomized, doubleblind, placebo-controlled clinical trial of varenicline. Drug Saf. 2010;33:289-301.

10. Campbell AR, Anderson KD. Mental health stability in veterans with posttraumatic stress disorder receiving varenicline. Am J Health Syst Pharm 2010;67:1832-7.

11. Harrison-Woolrych M, Maggo S, Tan M, Savage R, Ashton J. Cardiovascular events in patients taking varenicline. Drug Saf 2012;35:33-43.

12. Krebs P, Sherman SE. ACP Journal Club: review: varenicline for tobacco cessation does not increase CV serious adverse events. Ann Intern Med 2012; 157:JC2-2.

13. Sood A, Ebbert JO, Sood R, Stevens S. Complementary treatments for tobacco cessation: a survey. Nicotine Tob Res 2006;8:767-71.

14. White AR, Rampes H, Liu JP, Stead LF, Campbell J. Acupuncture and related interventions for smoking cessation. Cochrane Database Syst Rev 2011;(1): CD000009.
15. Orman, DJ, Margetis, D. The effects of auriculotherapy on smoking cessation. J Naturopathic Med 1991;2:41-2.

16. Antoniou D, Bouros D, Pavlakou G, et al. Aural electrical stimulation for smoking cessation: a double blind, placebo controlled study. Euro Resp J 2005; 26(Suppl):388s.

17. MacArthur Foundation. Depression management tool kit. Available from: http://www.depresion-primarycare. org/clinicians/toolkits. Accessed November 13, 2012.

18. Montalto NJ, Wells WO. Validation of self-reported smoking status using saliva cotinine: a rapid semiquantitative dipstick method. Cancer Epidemiol Biomarkers Prev 2007;16:1858-62.

19. Wu TP, Chen FP, Liu JY, Lin MH, Hwang SJ. A randomized controlled clinical trial of auricular acupuncture in smoking cessation. J Chin Med Assoc 2007;70:331-8.

20. He D, Medbo JI, Hostmark AT. Effect of acupuncture on smoking cessation or reduction: an 8-month and 5-year follow-up study. Prev Med 2001;33:364-72.

21. Oleson T. Auriculotherapy Manual: Chinese and Western Systems of ear Acupuncture. 2nd ed. Los Angeles, CA: Health Care Alternatives, Inc.; 1998.

22. Heatherton TF, Kozlowski LT, Frecker RC, Fragerström KO. The Fagerström test for nicotine dependence: a revision of the Fagerström Tolerance Questionnaire. Br J Addict 1991;86:1119-27.

23. DiClemente CC, Prochaska JO, Fairhurst SK, Velicer WF, Velasquez MM, Rossi JS. The process of smoking cessation: an analysis of precontemplation, contemplation, and preparation stages of change. J Consult Clin Psychol 1991;59:295-304.

24. Cohen S, Kamarck T, Mermelstein R. A global measure of perceived stress. J Health Soc Behav 1983; 24:385-96.

25. Toll BA, O’Malley SS, McKee SA, Salovey P, KrishnanSaran S. Confirmatory factor analysis of the Minnesota Nicotine Withdrawal Scale. Psychol Addict Behav 2007;21:216-25.

26. Velicer WF, Prochaska JO, Rossi JS, Snow MG. Assessing outcome in smoking cessation studies. Psychol Bull 1992;111:23-41.

27. Studts JL, Ghate SR, Gill JL, et al. Validity of selfreported smoking status among participants in a lung cancer screening trial. Cancer Epidemiol Biomarkers Prev 2006;15:1825-8.

28. Hollis $\mathrm{S}$, Campbell $\mathrm{F}$. What is meant by intention to treat analysis? Survey of published randomized controlled trials. BMJ 1999;319:670-4.

29. Graham JW. Missing data analysis: making it work in the real world. Annu Rev Psychol 2009;60:549-76.

30. Yeh ML, Chang CY, Chu NF, Chen JJ. A six-week acupoint stimulation intervention for quitting smoking. Am J Chin Med 2009;37:829-36. 